\title{
Editorial
}

\section{A PROCESS FOR PLANNING THE INTRODUCTION AND IMPLEMENTATION OF MULTIDRUG THERAPY FOR LEPROSY}

At first sight, the process outlined in this paper may appear to be unnecessarily complex. However, the introduction of multidrug therapy (MDT) into a pre-existing programme or the development of a new programme for MDT is not a simple matter. Since MDT is likely to be the last (and possibly best) chance we shall have, for the foreseeable future, to control leprosy and to cure individual patients, anyone who undertakes to use MDT is obligated to use it effectively; and this means careful planning.

The content of this document is based on the assumptions that there is a significant number of leprosy cases in the area under consideration, that a health service infrastructure exists, and that reasonable resources will be allocated to enable these cases to be detected and effectively treated.

For purposes of discussion the process is described under 11 different headings: 1 , context; 2 , the extent of the problem; 3 , overall goals; 4, obstacles; 5 , strategy; 6 , specific goals; 7 , activities; 8 , resources; 9 , schedules; 10, management systems (leading, organization, control, evaluation); 11 , management documentation (procedures, policies, job descriptions, records and reports, the clinical laboratory, the clinic, home visits).

\section{A Process for Planning Multidrug Therapy}

\section{CONTEXT AND NEED}

1.1 The first step is to define the boundaries (geographic and demographic) of the area in which the problem of leprosy is to be tackled. This might be a defined municipality or rural district with a known or estimated population and specific geographic boundaries; or much less satisfactorily, it might be simply those cases which are registered for treatment at a given centre and live within reasonable travelling distance of that centre.

1.2 Describe the existing leprosy control programme in the area and estimate its effectiveness. Include in the description all programmes which are offering diagnosis or care for leprosy, both government and private, for-profit and not-for-profit.

1.3 Collect data to define the Hansen's disease problem as accurately as possible: (i) known cases, characterized by age, sex, case type, deformity grading, treatment status (especially with respect to previous monotherapy treatment and the possibility of dapsone resistance); (ii) estimate total cases and state how this estimate has been derived; (iii) annual new cases registered by age, sex, case type, deformity grading and, if possible, means of diagnosis and time lapse between beginning of signs and symptoms and diagnosis over the previous 3-5 years; (iv) estimate of annual new cases in the area over the next 5 years. 
1.4 Describe the features of the area which are relevant to solving the leprosy problem. Include: existing health and social services, culture and attitudes to leprosy cases, and community resources that might be available for case-finding or patient care. Do not attempt to be exhaustive in this review of context. In reality it may only be possible to obtain rather scanty information - about leprosy prevalence - for instance, until a service has been started and patients begin to have confidence in it. Additional factors can be added to the contextual description as the need arises and opportunity occurs. Estimates of total cases (prevalence) and annual new cases (incidence) are likely to be inaccurate or not available at all.

\section{THE EXTENT OF THE PROBLEM}

Summarize the extent of the leprosy problem as revealed by the contextual review in terms of the numbers (case type, age, sex, and deformity grade) of cases, existing resources, facilities and community attitudes.

\section{GOALS FOR LEPROSY PROGRAMME}

Prepare general goals for the leprosy programme under the following headings:

3.1 Case-finding. Ninety-five percent of cases will be diagnosed and placed on chemotherapy before grade 2 deformity occurs.

3.2 Chemotherapy. Appropriate chemotherapy will have been prescribed at the time of diagnosis for all cases, and $90 \%$ of cases will continue with treatment, in accordance with medical instructions, until the prescribed treatment is completed. All cases with reaction will be identified and effectively managed in time to prevent deformity.

3.3 Care. All known cases will be provided with opportunities to receive appropriate care, and $75 \%$ of cases will cooperate with the care programme.

3.4 Surveillance. All post MDT cases with relapse or reaction will be detected and given appropriate chemotherapy.

\section{OBSTAClES}

Summarize the obstacles which are known to stand in the way of achieving the stated goals. Most of the important obstacles will be identifiable from the contextual analysis. Obstacles may be summarized under 3 headings:

4.1 Health services and health resources. These will be likely to include lack of trained staff, poor distribution of resources and negative staff attitudes to leprosy.

4.2 Obstacles in the patient community. Lack of confidence in existing official services, the existence of communities of beggars, reliance on traditional treatments, established patterns of irregular treatment, and numbers of institutionalized cases who fear any changes in the current system.

4.3 Community attitudes and culture. Ignorance of the truth about leprosy, ostracism of known cases, fear of infection and false perceptions of the human characteristics of cases.

\section{STRATEGY}

The general strategy for leprosy control and patient care is chemotherapy with 2 or more drugs, initiated as early as possible in the disease and continued for a limited time period.

\subsection{Case-finding strategy}

The following strategies, not all of which are equally practical, are available for case-finding in leprosy: 
A Voluntary presentation stimulated through effective care of existing cases, publicity concerning the availability of treatment and public education in the truth about leprosy.

B Referrals from other medical services.

C Active case-findings through surveys including:

(a) Contact surveys (contacts defined in terms of: (i) family relationships; (ii) household contacts; and (iii) work place contacts). (b) Special group surveys, including surveys of school children and workers. (c) Total population surveys.

The frequency and extent of surveys needs to be defined.

\subsection{Chemotherapeutic strategy}

This has 5 principal elements:

A A drug delivery system. This includes arrangements for the procurement, stocking, and inventory control of drugs; and decisions concerning the frequency of drug distribution to patients and the methods of drug distribution, drugs may be distributed either on an individual domiciliary basis or through clinic attendance.

B A patient categorization system. The same regimen is not given to all patients and a system is necessary for determining which regimen shall be given to which patient.

Each regimen needs to be defined in terms of the drugs used, the dose, the frequency and the duration of medication.

Each case needs to be defined in terms of: (a) Clinical classification, for instance, paucibacilliary or multibacilliary. Detailed criteria for determining classification on clinical, bacteriological, immunological, and histological grounds must be defined. (b) Definition of activity status, particularly in relation to previous treatment. A decision needs to be taken as to whether all cases should be treated or certain inactive cases should be excluded. Patients to be excluded need to be clearly defined; for instance, paucibaciliary cases, e.g. TT, lepromin positive $(8 \mathrm{~mm})$, who were never smear positive, had less than 20 skin lesions, and were treated with DDS monotherapy regularly for a total of not less than 4 years.

C A clinical and bacteriological review strategy. The frequency and requirements for clinical and bacteriological review must be prescribed, especially with respect to clinical records and quality control of bacteriology.

D Compliance checking. It is not enough to prescribe and provide treatment; arrangements must be made to check whether staff are complying with prescription instructions and patients are complying with the prescribed intake.

E Patient education. A strategy must be prescribed for patient education. There are a number of possibilities, including: (a) One-to-one education at the clinics; (b) appropriate literature for patients; (c) patient education through home visits; and (d) group education at clinics, including group discussions between patients.

\subsection{Patient care strategy}

Patient care is care in addition to chemotherapy. It is very important and mainly concerned with the prevention and correction of deformity and disability and in dealing with patient's social and economic problems. There are 4 main elements in a patient care strategy.

A Patient education in self-care.

B Programme assistance with self-care, for instance, provision of protective footwear.

C Programme provision of special care, for instance physical therapy, reconstructive surgery, vocational training.

D Social services, including assistance with family problems, personal psychological problems, and with economic problems. 


\subsection{Surveillance strategy}

The purposes of post-MDT surveillance are to detect and retreat relapsed cases and to detect any late manifestations of reaction or side-effects of the drugs used. Three basic strategies are possible:

A Rely on voluntary reporting by patients: reinforced by more patient education and made as easy as possible by the provision of acceptable clinics.

B Insist on patients continuing to attend the clinic for review at specified intervals and for a specified duration: back this up by home visits to follow-up absentees.

C Arrange home visits by trained staff to undertake surveillance in the patients' homes.

\subsection{Strategy implementation}

Decisions must be taken as to who is to provide these services, and where they are to be provided. A number of options are available including:

A A vertical leprosy programme in which all aspects of leprosy control and patient care are undertaken within the programme.

B A vertical leprosy programme with referral of cases to special services for special needs, e.g. reconstructive surgery, vocational training, social services.

C An integrated programme in which general medical services accept responsibility for casefinding, chemotherapy and patient care at the same level as with other chronic diseases, and have access to specialist referral services for special problems.

Each strategy has technical, financial and sociological implications. Patients preferences need to be seriously considered.

\section{SPECIFIC GOALS}

The role of the planner at this stage is to define the goals which must be reached in order to implement the strategy outlined in Section 5.

The details of the goals will necessarily depend to some extent on the strategies adopted. In the following example 3 sets of assumptions have been made concerning strategy:

A Diagnosis, contact survey, drug delivery, patient care and surveillance will take place mainly in special leprosy clinics, i.e. it will be a vertical programme. Patients will be expected to attend a clinic once each month.

B Staff will be available to visit patients at home in order to check on compliance, reinforce patient education, follow-up absentees, and supplement clinic-based contact surveys.

C Patient and public education will be the primary case-finding strategy but in addition efforts will be made to discover cases through: 1 , referrals from other health services; 2 , school surveys; and 3 , total population surveys in selected areas.

\subsection{Goals for case-finding}

Overall goal: $95 \%$ of cases will be diagnosed and placed on chemotherapy before Grade II deformity occurs.

Specific goals are as follows:

\section{A Problem identification}

1 All registered patients will be able to describe and recognize cardinal signs and symptoms of leprosy and its reactions.

2 All medical personnel responsible for making diagnosis in government and private clinics in the area will have been provided with a diagnostic guide to leprosy; information concerning timing, location and telephone numbers of the leprosy clinic and, in addition, they will be aware of the possibility of cure of leprosy and of the necessity for early diagnosis and chemotherapy. All medical 
personnel will cooperate by referring cases they recognize to the leprosy clinic for diagnosis and treatment.

3 All leprosy clinic staff, and all engaged in leprosy patient home visitation, will recognize patients with cardinal signs and symptoms of leprosy and/or reaction and will refer patients appropriately.

\section{B Problem solving}

1 All diagnosed cases will have case notes appropriately completed on the prescribed forms. These records will include data to support diagnosis, classification, deformity grading and activity status.

2 All multibacillary cases will be smeared. All cases of doubtful classification will be smeared. Not more than 5-15\% of negative cases will be smeared. All indeterminate cases will be biopsied before diagnosis is confirmed. (If resources permit all cases will be biopsied.)

3 All household, family and work contacts of leprosy cases will have been examined clinically within 1 year of the case being registered. Examination for contacts of smear positive cases will be repeated annually for 5 years.

4 All children attending school will be able to describe early signs and symptoms of leprosy.

5 Ninety percent of adults in the community will have been made aware of the possibility of leprosy through the mass media and will be able to describe cardinal signs and symptoms.

\subsection{Chemotherapy and management of reactions}

Overall goal: Appropriate chemotherapy will have been prescribed at the time of diagnosis to all cases. Ninety percent of cases will take treatment according to prescription. Ninety percent of reactions will be recognized and appropriately managed before any increase in deformity ensues.

\section{A Treatment goals}

1 Ninety percent of paucibacillary cases will have completed supervised multidrug therapy treatment within 9 months of registration.

2 Ninety percent of multibacillary cases will have attended regularly for supervised treatment.

3 Ninety percent of $\mathrm{MB}$ and $\mathrm{PB}$ cases will have been at least $90 \%$ compliant with treatment instructions with regard to drug intake.

\section{B Service goals}

1 Supplies (drugs, record systems, laboratory supplies) will have been made available in adequate quantities and appropriate timing at each clinic.

2 Staff will make appropriate prescriptions and record attendance and drugs issued at each attendance.

3 Staff will review cases and record findings at each attendance, to detect side-effects of drugs and the clinical evidence of resolution and reaction.

4 Staff will manage reaction appropriately through patient education, treatment and/or referral.

5 Staff will take, read, record, and interpret the results of smears.

6 Clinics will have been run at advertised times and places.

7 Patients will appreciate the importance of regular attendance and regular drug intake.

8 Patients will recognize signs and symptoms of reaction and side-effects of drugs.

9 Interpersonal relationships between clinic staff and patients will be such as to encourage regular attendance. Patients will have confidence in the integrity, concern and reliability of staff.

\subsection{Goals for care}

Overall goal: All known cases will be provided with appropriate opportunities to receive care.

Special goals: 1 All patients will be able to undertake appropriate self care. 
2 All patients in need will have been provided with footwear and other devices, and will use them.

3 All cases likely to benefit from reconstructive surgery, according to the criteria defined by Fritschi, will have the opportunity of accepting reconstructive surgery at an appropriate time.

4 Patients in need, according to defined criteria, will have received appropriate social and economic support.

5 Patients in need, and likely to benefit according to defined criteria, will have received appropriate job training and placement.

\subsection{Goals for surveillance}

Overall goal: All post-MDT relapsing and reacting cases will be detected and given appropriate treatment.

Specific goals: 1 Ninety percent of treated MB cases will attend for surveillance once each year for 5 years within 1 month of the due date.

2 Ninety percent of treated PB cases will attend for surveillance 6 months after completion of the chemotherapy and subsequently once each year for 5 years, within 1 month of the due date.

3 Ninety percent of cases who fail to attend for surveillance will be visited and examined at home within $3 / 12$ of the due date (provided social considerations do not preclude home visits).

4 Staff will correctly identify fresh clinical or laboratory signs of leprosy as relapse or reaction in $90 \%$ of cases, and provide appropriate treatment.

5 Appropriate records will be çompleted on all cases surveyed.

\section{ACTIVITIES (TASKS)}

In order to reach each of the goals identified in step 6, certain activities are needed. The role of the planner is to identify these activities.

Activities may be most readily categorized into 9 groups or functions:

\subsection{Clinic function}

Clinic activities are determined by the need for patients to have access to services within reasonable reach of home. The role of the planner is to insure that the clinic is appropriately located, equipped, and staffed to carry out the following activities:

A Take case histories, and undertake clinical examinations and (order) special investigations for the following purposes: (a) new cases: diagnosis, classification, determination of deformity grade, activity status, presence or absence of reaction, treatment status, existence of other problems; (b) review cases: determine clinical progress, deformity grade, activity status, presence or absence of reaction, treatment status, side effects of drugs, resistance or other problems; (c) surveillance and care cases: determine deformity grade, activity status, presence or absence of reaction, presence or absence of relapse, existence of other problems. (d) All cases: prepare or review list of contacts.

B Initiate appropriate action, namely: (a) prescription and issue of drugs; (b) patient education; (c) referral for special services, e.g. physical therapy, occupational therapy, surgery, social and/or economic assistance; (d) arrange for repeat visits, arrange for home visits, arrange for contact examinations; (e) make appropriate records: clinical records, clinic register; ( $)$ prepare appropriate reports.

\subsection{Domiciliary function}

There are 6 basic activities to be undertaken during home visits as follows:

A Patient encouragement and education. 
B Family education.

C Compliance, encouragement and review.

D Self-care, encouragement and review.

E Clinical examination of contacts (and surveillance cases).

F Social and economic needs review.

\subsection{Health facility function}

There are 4 basic activities for general health facilities, namely:

A Recognition of leprosy cases, including diagnosis, reactive phenomena, and relapse.

B Referral of leprosy cases to the appropriate clinic.

C Management of other diseases occurring in leprosy cases.

D Specialist medical services for leprosy cases.

\subsection{Laboratory function}

The following laboratory activities are necessary:
A Smear taking, reading, reporting.
B Biopsy taking, reporting.
C Lepromin testing, administration reading and reporting.
D Serology venesection, and storage.
E Routine laboratory tests, determination of haemoglobin, etc.

\subsection{Public education function}

The following activities comprise the public education function:
A Ascertainment of existing knowledge, attitudes and practices with respect to leprosy.
B Identification of community leaders.
C Determination of specific goals for public education.
D Involvement of community leaders in planning public education.
E Implementation of public education through public meetings (drama discussion, talks, etc.), mass media radio and television, newspapers and other literature.
F Education of special groups: schools, churches, women's clubs, etc.
$G$ Review of the effectiveness of public education in changing knowledge, practice and attitude.

\subsection{Survey function}

Properly conducted total population surveys are an effective, but often costly, method of casefinding. Poorly conducted surveys are both ineffective and inefficient. Survey activities include the following:
A Planning and preparation of surveys, and training of the survey team.
B Delineation and mapping the area to be surveyed.
C Taking a census and population education.
D Conducting the survey; household census, clinical examination, recording absentees, arranging follow-up visits to examine absentees, advice or referral of incidental cases with other diseases.

\subsection{Rehabilitation function}

A full range of rehabilitation activities is listed below, although it is recognized that many of these will only be required with respect to a small proportion of patients and some may not be available at all. The rehabilitation activities are as follows:

A Assessment of need and referral to the appropriate service. 
B Additional patient education in self-care.

C Provision of footwear and other aids.

D Physical therapy.

E Reconstructive surgery.

F Social assistance.

G Economic assistance.

$\mathrm{H}$ Assistance with work, i.e. job training and placement.

\subsection{Training function}

As soon as programme activities have been allocated to designated staff, steps can be taken to train staff to undertake these activities. The main training activities comprise the following:

A Determination of existing skills and knowledge in the staff.

B Preparation of training objectives.

C Preparation of training assessment instruments.

D Preparation of a training programme.

E Obtain or prepare training materials.

F Conduct training.

G Evaluation of effectiveness of training.

With respect to multidrug therapy, particular attention is to be paid to training clinical staff in accurate recording of clinical findings, accurate classification, and smear interpretation, appropriate decisions regarding prescription and continuation or discontinuing treatment, and recognition of relapse or reaction in cases that have discontinued chemotherapy. Laboratory staff will also probably require additional training and the introduction of a quality control method for smear taking, staining, and reading.

\subsection{Management function}

Management has been defined as all activities for which no one else has been given responsibility. The specific activities of management will include the following:

A Preparation of protocols, processes, record and reporting systems for the activities listed above.

B Preparation of job descriptions.

C Budgeting.

D Staffing.

E Procurement and distribution of supplies.

F Provision of transportation.

G Control of activities.

$\mathrm{H}$ Leadership and motivation.

I Public relations and relationships with other health services.

J Evaluation.

\section{RESOURCES}

The actual details of estimates of the resources needed for implementation of multidrug therapy vary widely from situation to situation. The most important factors in this variation include: (a) The local economic level particularly with respect to salaries and cost of transportation; (b) population density; (c) endemicity of leprosy; (d) case type rates in the leprosy population proportion of cases with disability; and (e) the extent to which general medical services take responsibility for leprosy patients. However, some general statements can be made:

A Cost of drugs. Paucibacillary cases (PB): US\$2.1-2.77 per patient per course. (Assume $98 \%$ of cases required 1 course only). 
Multibacillary cases (MB): US\$23 per patient per year. (The average multibacillary case is likely to require 3-4 years of treatment.)

B Relapses. It may be assumed that not more than 1-2\% of paucibacillary and/or multibacillary cases will relapse.

C Footwear costs. The proportion of cases requiring footwear vary very widely from 10-30\% or more. Similarly the costs of footwear vary from US\$5 to $\$ 10$ or more per patient needing footwear per year.

D Staff workload. The first 1 to 2 years after the introduction of multidrug therapy into an existing leprosy treatment programme is likely to double the manpower requirements for chemotherapy and care. There are several reasons for this of which the most important are the introduction of supervised treatment, the higher standards required for clinical examination and recording, the need for extensive patient education, and the likelihood that increased numbers of cases will come forward once MDT is introduced. However, after 1 to 2 years there will be a very substantial reduction in the numbers of paucibacillary cases under care and this is likely to result in a reasonably substantial reduction in workload. Staff workload is likely to fall to the level it was before MDT by the end of the 2nd year.

E Laboratory work. A good laboratory technician can process not more than 20 slides per day. The actual workload imposed upon the laboratory will depend upon policy decisions regarding the frequency of smears (for instance 1 smear per year) and the requirements for smearing clinically paucibacillary cases. It is important to note that it takes longer to read a negative smear than a known positive smear.

F Domiciliary services and surveys. The costs of visiting patients at home and carrying out total population surveys are very much influenced by population density, leprosy endemicity, pay scales and the cost of transportation. The number of homes visited per worker per day may be as few as 2 to 3 or as many as 20 to 30 , and the average number of individuals visited per home also varies widely.

G Hospital admissions. Experience has shown that the provision of 10 beds per 1000 registered cases is a very comfortable provision for leprosy and its complications. It should be noted however that up to $75-80 \%$ of these admissions will be for ulcer care. The actual number of admissions required for acute complications of leprosy in a well organized control programme is likely to be less than $5 \%$ of total patient load per year and to need a maximum of 1 or 2 beds per 1000 patients.

H Transportation. Mobility to enable staff to reach patients and to enable patients to have access to services is an essential part of an effective leprosy control programme. The costs of mobility are usually underestimated.

The need for programme transportation varies enormously and is dependent upon population density, endemicity of leprosy, availability of roads and availability of public transportation to leprosy patients.

\section{SChEDUles}

Scheduling the introduction of MDT and its implementation clearly depends upon the local situation. Some general remarks may be helpful:

A Recognize the lapsed time between ordering and securing drug supplies. It is disastrous to start a programme and run out of drugs.

B Do not underestimate the staff workload which will result from the need to review existing cases and update the clinical and laboratory records.

C Programmatic changes which affect patient behaviour should not be introduced in too rapid a sequence. Patients need time to adapt to new situations.

D Staff training should be undertaken as close to the time of utilization of training as possible.

E If the programme being planned is a very large one, it is almost certainly worthwhile undertaking a small scale tryout before embarking on the total programme. 
In any programme it is necessary to make arrangements for organization, for leadership, and for controlling activities.

A Organization. The purpose of organization is to allocate tasks into groups so as to make coordination possible. A wide variety of groupings of the tasks (activitics, identified in Section 6) is no doubt possible and in actual practice the groupings chosen will depend on: (a) Access and transportation. That is the need for a patient to have physical access to services at reasonable convenience and cost; (b) traditional delineation of the roles and competencies (job descriptions) of existing staff; and (c) the location of existing services, including services which do not at present offer any service to leprosy patients but may be required to do so.

Important principles are to ensure that an appropriate individual is given responsibility and resources necessary to carry out each activity listed and to ensure that each individual given responsibility is responsible only to one supervisor for a given activity. Organization should be kept as simple as possible.

B Leadership. The increased workload and the increase quality of work which is consequent upon the introduction of MDT and necessary for its success places great demands upon programme leadership. Programme leaders at least need to be aware of this and committed to it before embarking upon implementation of multidrug therapy.

C Control. Three fundamental changes in control systems are needed in most leprosy control programmes if MDT is to be effectively implemented. These are: (a) the introduction of cohort recording and analysis for the control of chemotherapy, particularly chemotherapy for paucibacillary cases; (b) the introduction of quality controls for clinical records, classification, deformity grading, and laboratory work; (c) the introduction of compliance checking for drug intake.

\section{MANAGEMENT DOCUMENTATION}

A Programmes, Goals, and Strategies (see Sections 3, 5 and 6).

B Clinical documentation:

Description of the regimen, (WHO Technical Report Series 675 (1982) (OXFAM Practical Guide No. 3, pp. 4, 5, and 6).

Definition of cases to be given MDT - Classification of cases into MB and PB (WHO TRS 675, 24);

Definition $6 f$ previously treated cases to be excluded from MDT ( $O P G 3$, pp. 4-6);

Description of the side-effects of the regimen [Jopling, Lepr Rev, 54: 270 (1983)].

Instructions for prescriptions including: Paucibacillary-time limits for the completion of treatment, and management of cases that voluntarily interrupt treatment ( $O P G 3$, p. 12), treatment of relapse cases;

Multibacilliary-definition of a regular case, definition of an irregular case, cut-off point for treatment, and management of cases that voluntarily interrupt treatment $(O P G 3, \mathrm{p} .12)$, and treatment of relapse cases.

Recognition of relapse - distinguishing relapse from reaction (OPG 3, pp. 13-19):

Management of reaction;

Recognition of reaction;

Basic procedures;

Action to be taken when treatment of reaction overlaps with termination of MDT.

Instructions for surveillance (OPG 3, pp. 10-12).

Clinical records:

(Classification, deformity grading, reactive phenomena.)

Treatment records:

Clinic retained records; 
The treatment register;

Patient retained records.

C Reporting: Indicators of effectiveness (ILEP Ad Hoc Working Group No. 1, 1985):

Reporting systems (all from ALERT 1987):

The central register; The quarterly report; The annual report; Paucibacilliary cohort report; Multibacilliary completion report; Transfer and referral; Release from treatment.

D Smears:

Methodology (Technical guide for smear examination for leprosy by direct microscopy. Royal Tropical Institute, Amsterdam, 1983).

E Quality control for smears (OPG 3, pp. 26-28), procedures:

Instructions for conducting a clinic;

Instructions for a home visit;

Prescription of footwear;

Staff job descriptions;

Programme organization chart.

F Control systems:

Activity controls - timetables, schedules, diaries, gantcharts;

Financial controls;

Supplies controls - standard stock, inventory control systems;

Transportation-acquisition and control of transport.

G Surveys:

a Preparation conduct of school and smear group surveys;

b Preparation and conduct of total population surveys.

H Compliance checking:

a Pill counting.

b Tests for the presence of DDS in urine (Huikeshoven H. 'Multi-Center Evaluation of a Spot Test for Detection of Dapsone in Urine', The Star, 1984, vol. 43.4, pp. 12-13).

American Leprosy Missions

W F Ross

One Broadway

Elmw'ood Park

New Jersey 07407

USA 


\section{Special Programme for Research and Training in Tropical Diseases; Eighth Report 1987}

This is a strongly bound paperback of 191 pages entitled 'Tropical Disease Research; a Global Partnership'. It is the eighth programme report for the UNDP/World Bank/WHO Special Programme for Research and Training in Tropical Diseases (TDR) with a Foreword by the Director-General, Dr Halfdan Mahler, and an introduction by Dr Tore Godal, Director of TDR. Progress in all 6 of the diseases chosen (malaria, schistosomiasis, filariasis, leprosy, leishmaniasis and trypanosomiasis) is described in detail, using terminology which is sometimes technical, but for the most part general and highly readable. The account of leprosy in section 8 , page 115 , is brief but comprehensive, giving an exceptionally good account of the present situation, the main problems, progress in diagnosis, chemotherapy and vaccine development. In the final paragraphs, TDR's future priorities for leprosy research are described as follows:

Immunology. Continued field trials on antileprosy vaccines and the use of data from these trials for epidemiological studies, including those undertaken to assess new serological and other diagnostic tests; maintenance of armadillo colonies for large-scale production of $M$. leprae; DNA sequencing of $M$. leprae gene fragments, identification of the complete genes and expression of these genes in foreign host microorganisms with a view to large-scale production of expressed antigens and their application to research on diagnostic tests, immunization and drug development; identification of $M$. leprae genes involved in protective immunity; development of techniques for detecting $M$. leprae antigens in clinical specimens; development of specific serological assays and skin tests based on synthetic peptides; and development of methods for genetic engineering of BCG with a view to its use as a vehicle for protective antigens in vaccines against leprosy and other diseases;

Chemotherapy. Studies on the efficacy, acceptability and operational feasibility, in both lepromatous and nonlepromatous leprosy, of multidrug therapy regimens of even shorter duration than those in current use; development of new drugs through synthesis and screening activities based on leads from other diseases and on 'molecular modelling'; validation and improvement of the rapidity, sensitivity and specificity of in vitro drug screening systems; evaluation of the impact of multidrug therapy on leprosy transmission; new approaches (including inoculation of nude mice and specific antigen assays) to the monitoring of chemotherapy; and development of effective, nonsteroidal, non teratogenic drugs to prevent and control leprosy reactions and nerve damage.

\section{Fortieth World Health Assembly: towards Elimination of Leprosy}

Recalling resolution WHA32.39 and previous resolutions of the Health Assembly and the Executive Board regarding leprosy. Noting: a, the increasing commitment of several Member States to eliminate leprosy as a public health problem in their countries, as part of their goal of health for all by the year 2000; b, the significant progress made in recent years in leprosy treatment, including the use of new drugs in multidrug therapy, which has made leprosy treatment far more effective; $c$, the very promising research advances being made towards the development of early diagnosis, immunology and vaccines, leading to effective leprosy prevention programmes; $\mathrm{d}$, the increasing role being played by nongovernmental organizations in leprosy control:

1 Urges Member States with endemic leprosy: 1, to allocate adequate priority to and resources for leprosy control within their public health services as part of primary health care; 2 , to strengthen health education through the media and community participation with a view to overcoming the stigma and phobias traditionally associated with the disease in many societies, and to institute adequate legal guarantees protecting the rights of cured leprosy patients; 3 , to provide improved training in leprosy for health workers of all categories, and especially those working in the field of leprosy, to ensure early case-finding, accurate diagnosis, and the implementation of multidrug therapy programmes; 4 , to institute active programmes, including research, for the rehabilitation of leprosy patients who have acquired disabilities and deformities; 5 , to work out a system of awards, prizes and rewards for outstanding contributions to leprosy control and research.

2 Requests the Director-General: 1, to continue the successful technical and scientific guidance to Member States and to support their multidrug therapy programmes for leprosy control; 2, to intensify the Organization's activities in leprosy control by additional mobilization and coordination of scientific and material resources directed at implementing multidrug therapy, rehabilitation and training; 3, to strengthen support for the development of more effective tools against leprosy through multidisciplinary research in both the natural and social sciences; 4 , to intensify the search for improved drugs and vaccines through the Special Programme for Research and Training in Tropical Diseases; 5, to promote further the partnership approach between nongovernmental organizations, Member States and WHO to achieve leprosy control and rehabilitation where necessary; 6, to keep the Executive Board and the Health Assembly informed of the progress made.

Twelfth plenary meeting, 15 May 1987 A40/VR/12. WHO, Geneva. 